\title{
Scarless repair of acute and chronic kidney injury in African Spiny mice (Acomys cahirinus)
}

Daryl M. Okamura ${ }^{1,3,{ }^{*}, \#}$, Chris M. Brewer ${ }^{2,3,{ }^{*}}$, Paul Wakenight $^{4}$, Nadia Bahrami $^{3}$, Kristina Bernardi $^{3}$, Amy Tran ${ }^{3}$, Jill Olson $^{3}$, Xiaogang Shi $^{3}$, Adrian M. Piliponsky ${ }^{1,2,5}$, Branden R. Nelson ${ }^{4}$, David R. Beier ${ }^{1,3,}$ Kathleen J. Millen ${ }^{1,4, \#}$, Mark W. Majesky ${ }^{1,2,3,6, \#}$

1 Department of Pediatrics, University of Washington, Seattle, WA, USA

2 Department of Pathology, University of Washington, Seattle, WA, USA

3 Center for Developmental Biology \& Regenerative Medicine, Seattle Children's Research Institute, Seattle, WA

4 Center for Integrative Brain Research, Seattle Children's Research Institute, Seattle, WA

5 Center for Immunity \& Immunotherapies, Seattle Children's Research Institute, Seattle, WA

6 Institute for Stem Cell \& Regenerative Medicine, University of Washington, Seattle, WA

${ }^{*}$ Authors contributed equally to this work.

\#Correspondence to:

Daryl M. Okamura, Center for Developmental Biology \& Regenerative Medicine, Seattle Children's Research Institute, University of Washington, Seattle,

WA. daryl.okamura@seattlechildrens.org 
Kathleen J. Millen, Center for Integrative Brain Research, Seattle Children's Research Institute, University of Washington, Seattle, WA. kathleen.millen@seattlechildrens.org

Mark W. Majesky, Center for Developmental Biology \& Regenerative Medicine, Seattle Children's Research Institute, University of Washington, Seattle, WA. mwm84@uw.edu

\section{Summary}

Solid organ fibrosis is a major burden on global health and medical care costs. Muroid rodents of the genus Acomys (African Spiny mice) are terrestrial mammals that evolved remarkable abilities to regenerate severe skin wounds without scar formation. However, whether scar-free wound repair in Acomys extends beyond skin to vital internal organs is not known. Here, we used two aggressive kidney injury models known to produce severe renal fibrosis and show that despite equivalent acute kidney injury, there was rapid restoration of nephron structure and function without fibrosis in Acomys compared to extensive fibrosis leading to renal failure in Mus musculus. These results suggest Acomys species have evolved genomic adaptations for wound healing that activate regenerative repair pathways not only in skin, but also in vital internal organs. Our findings have important implications for discovering a long-sought evolutionary solution to internal organ injury and regeneration. 


\section{Introduction}

Solid organ fibrosis is the result of chronic inflammatory processes and dysregulated wound healing that leads to progressive loss of tissue function and eventual organ failure. ${ }^{1}$ The global health care burden for cumulative loss of vital organ function due to progressive fibrosis is enormous. ${ }^{2}$ There are currently very few treatment options for patients with end stage renal disease or similar degenerative fibrotic conditions in the heart, lung, liver, or other critical internal organs. ${ }^{3}$ Looking to nature for a possible solution, it was reported that adult rodents of the genus Acomys (African spiny mice) can shed their dorsal skin as a deterrent to avoid predators and fully regenerate the lost tissue without fibrosis or tissue overgrowth. ${ }^{4}$ The restored skin is complete with hair follicles, sebaceous glands, cartilage, adipose tissue, nerves, and blood vessels in the correct architectural organization. ${ }^{5,6}$ While this remarkable wound healing response in the skin has been examined in some detail, the important question of whether or not scarless regenerative wound repair in Acomys species extends beyond skin to vital internal organs remains unanswered.

In the experiments reported here, our objective was to produce injuries to Acomys kidney that are known to promote severe fibrotic responses leading to organ failure in murine kidney. Our goal was to determine whether or not scarless, regenerative wound healing observed in Acomys skin extends to critical internal organs. We now provide the first reported evidence that scarless wound repair first observed in the skin does indeed extend to a major internal organ in the African spiny mouse. We demonstrate that in two aggressive models of kidney disease, unilateral ureteral obstruction (UUO) and ischemia reperfusion injury (IRI), there was a near complete absence of fibrosis and a rapid regeneration of nephron function in A. cahirinus. By contrast, paired groups of $M$. musculus (outbred CD-1 or inbred C57BL/6J) developed severe kidney fibrosis that rapidly progressed to complete renal failure. These studies represent the first step in an evolutionary approach to understand how mammalian wound healing can be 
uncoupled from the fibrotic response to injury and redirected toward regeneration of complex organ function in mammals.

\section{Results}

\section{A. cahirinus fails to develop fibrosis after UUO injury}

Tubulointerstitial fibrosis is the final common pathway of many forms of kidney disease..$^{1,3,7}$ Unilateral ureteral obstruction (UUO) is a reliable and aggressive model of chronic kidney injury and robust interstitial fibrosis. In previously reported studies where the contralateral kidney was removed after $7 d$ of obstruction in M. musculus, UUO kidneys were found to have about $50 \%$ function. After $14 \mathrm{~d}$ they become nonfunctional resulting in $100 \%$ mortality from kidney failure. ${ }^{8}$ We performed UUO surgeries on $A$. cahirinus and $M$. musculus (outbred CD-1 and inbred C57BL/6J (B6) strains were used) and retrieved injured kidneys (UUO) and contralateral kidneys (NK) at the times indicated in Fig 1. We found that even after 14d of obstruction with obvious signs of hydronephrosis (Fig 1A,B), the gross anatomic structure and parenchymal thickness (between arrows, Fig $\mathbf{1 C}$ ) were remarkably preserved in $A$. cahirinus compared to $M$. musculus (B6). This preservation of tissue structure was confirmed by the maintenance of relatively normal kidney weights (UUO/NK) in obstructed $A$. cahirinus kidneys compared to rapid declines in kidney weight in M. musculus as a a result of progressive fibrosis (ratio of slopes: $m_{\text {Mus }} / m_{\text {Acomys }}=-7.5 ; p=0.03$, Fig $\left.1 \mathbf{C}\right)$. There were no significant differences in uninjured contralateral kidney weights between $A$. cahirinus and M. musculus (data not shown).

Progression of fibrosis was monitored by three different assays. Total collagen levels were measured as hydroxyproline content per wet kidney weight. Kidney collagen levels increased rapidly in $M$. musculus while $A$. cahirinus exhibited no significant change from the uninjured contralateral kidney (NK) ( $n=6-8$, Fig 1D). Remarkably, even out to 21d of obstruction, there were no significant differences in total collagen levels between UUO kidney and uninjured 
contralateral kidney (NK) in A. cahirinus (Fig 1D; Acomys:NK vs D3-21, NS). Computerassisted image analysis of picrosirius red-stained kidney tissue sections demonstrated a nearly complete absence of interstitital matrix fibrosis at each time point after UUO injury in $A$. cahirinus even out to $21 \mathrm{~d}$ of obstruction (Acomys: NK vs D3-21, NS), compared to extensive fibrosis in M. musculus kidneys (Fig 1E, Supp Fig 1). Interstitial fibrosis, inflammation, and tubular atrophy (IFTA) were blindly scored on Masson's trichrome stained sections. We found that IFTA scores were markedly reduced in A.cahirinus compared to M. musculus (B6) despite chronic obstructive injury in both species (Fig 1F, Supp Fig 2). In order to test our findings in an outbred strain of M. musculus, we performed UUO surgeries on CD-1 mice and measured fibrosis severity by picrosirius red staining. Of interest, we found even more dramatic increases in interstitial fibrosis in CD-1 mice producing even greater differences in fibrotic tissue areas when compared to $A$. cahirinus (Fig $\mathbf{G}, \mathbf{H} ; p<0.0001$ ). All together, these results demonstrate that, in contrast to either inbred or outbred M. musculus strains, $A$. cahirinus does not develop fibrotic tissue in response to severe chronic obstructive kidney injury.

\section{A.cahirinus maintains tubular integrity and modifies myofibroblast accumulation after UUO injury}

In order to quantify the degree of obstructive tubular injury, we measured the dilated tubular area in $A$. cahirinus and $M$. musculus (B6) at $3 d$ through $14 d$ after UUO. As expected from the obvious hydronephrosis seen in Fig 1A,B, we found that tubular dilation increased significantly in both species following UUO compared to the contralateral uninjured kidney, and peaked at $7 \mathrm{~d}$ (Fig 1I; $p<0.05$ ). Importantly, there were no significant differences in the extent of tubular dilation between $A$. cahirinus and M. musculus at any of the time points examined (Fig $\mathbf{1 I}$ ). Activated myofibroblasts (positive for smooth muscle a-actin, Acta2) are a significant source of collagen-rich extracellular matrix produced during kidney fibrosis. Chronic tubular injury is known to promote the production of intrarenal profibrotic cytokines that activate myofibroblasts. ${ }^{9}$ 
While Acta2 immunolabeling increased after UUO in both species, M. musculus exhibited higher levels of Acta2+ myofibroblasts compared to A. cahirinus (Fig $2 \mathrm{~A}, \mathbf{B} ; \mathbf{p}<0.01$ ). In contrast to the lack of significant fibrosis in A. cahirinus after UUO (Fig 1D,E), there was a significant increase in Acta2+ myofibroblasts at D14 after UUO compared to both NK and D3 time points (Fig 2B, $p<0.05)$. These results suggest that the absence of interstitial matrix deposition in $A$. cahirinus after UUO injury is not due to the absence of myofibroblasts.

Chronic inflammation with a predominance of macrophages is a characteristic finding in organ injury and is strongly correlated with tissue fibrosis. ${ }^{10}$ In order to quantify macrophage infiltration, whole kidneys were enzymatically digested into single cell suspensions and analyzed for F4/80 expression by flow cytometry (Fig 2C,D). As expected, the number of F4/80 macrophages increased in M. musculus UUO kidneys with advancing obstruction (7d and 14d) compared to contralateral normal kidneys $(p<0.001)$. In $A$. cahirinus, increases in macrophage content were both delayed and diminished over the same time course (Fig 2D). Similar to Acta2 data from $A$. cahirinus kidneys, there was a significant increase in $\mathrm{F} 4 / 80+$ macrophages at D14 compared to NK and D3 time points (Fig 2D, p<0.001). In comparing $A$. cahirinus with M. musculus kidneys, significant reductions in F4/80+ macrophage content were found at each time point examined $(p<0.05)$ but less dramatic than the fibrosis $(F i g$ 1D,E) and myofibroblast (Fig 2B) data. These results suggest that the unique absence of fibrosis in $A$. cahirinus is not due to a complete absence of a chronic inflammatory response or an absence of myofibroblasts in injured kidney tissues but suggests an evolutionary adaptation in wound repair.

Tubular integrity is strongly correlated with nephron function and can serve as a histological surrogate of whole kidney function. ${ }^{11,12} \mathrm{Cdh1}$ (E-cadherin) is an indicator of tubular cell integrity and polarity whose expression is lost with ongoing obstructive injury leading to loss of tubular architecture. $^{13}$ As expected for M. musculus there was progressive loss of Cdh1 expression 
with each time point after UUO compared to the contralateral normal kidney (Fig 2E,F). However, in $A$. cahirinus, there were no significant changes in Cdh1 protein levels with advancing obstructive injury until D14 compared to the contralateral uninjured kidney. There were no differences in Cdh1 expression levels in uninjured contralateral kidneys between $A$. cahirinus and M. musculus (Fig 2F). Thus, despite severe chronic obstruction, Cdh1 protein levels were maintained in $A$. cahirinus while becoming significantly decreased in $M$. musculus injured kidneys with each time point (Fig 2F). All together, these results demonstrate that despite equivalent tubular dilation with obstruction (Fig $\mathbf{1 K}$ ), we found significant attenuations in myofibroblast activation, macrophage infiltration, and loss of Cdh1 in A. cahirinus kidneys that were correlated with preservation of tubular integrity and lack of interstitial fibrosis compared to M. musculus.

\section{Renal fibrosis is reduced in A. cahirinus despite equivalent ischemic injury}

Although the UUO model is useful in the study of renal fibrosis, it is not directly translatable to human kidney disease. By contrast, ischemic kidney injury is one of the most common causes of acute kidney injury in humans. We sought to test our findings in a second model of severe kidney injury with unilateral ischemia-reperfusion injury (uni-IRI) following prolonged 40 min of ischemia. One of the questions arising from our results with the UUO model was whether $A$. cahirinus was able to resist acute kidney damage rather than alter the subsequent fibrogenic cascade. Therefore, we performed uni-IRI with a simultaneous contralateral nephrectomy (uni$\mathrm{IRI}+\mathrm{Nx}$ ) on $A$. cahirinus and $M$. musculus and sacrificed them at $24 \mathrm{~h}$ after surgery in order to correlate kidney function with histology after severe acute injury. We found a significant elevation in blood urea nitrogen (BUN) levels $24 \mathrm{~h}$ after uni-IRI+Nx in both $A$. cahirinus and $M$. musculus (Fig 3A). Importantly, these acutely elevated BUN levels were not significantly different between species (Fig 3A). In fact, there was a trend towards higher BUN levels in $A$. cahirinus (BUN: Acomys vs Mus, $129 \pm 24$ vs $102 \pm 16 \mathrm{mg} / \mathrm{dL}$ ). H\&E sections on the Uni-IRI-Nx 
kidneys from $A$. cahirinus and M. musculus at $24 \mathrm{~h}$ were analyzed for tubular cell necrosis, casts, and dilation and assigned cumulative tubular injury scores. Consistent with our kidney function data, we found no differences in tubular injury scores at $24 \mathrm{~h}$ between $A$. cahirinus and $M$. musculus (Fig 3B,C) and therefore conclude that both species experience equivalent levels of acute ischemic injury and tissue damage after prolonged renal ischemia as assessed both histologically and functionally.

In order to investigate the ability of $A$. cahirinus to repair acute kidney injury, we performed uniIRI without contralateral nephrectomy to allow long term survival in both species and then sacrificed animals at $14 d$ to assess kidney structure by histology. Remarkably, despite severe acute ischemic injury, we found a near complete absence of fibrosis by picrosirius red staining, and a robust preservation of renal mass, in $A$. cahirinus compared to $M$. musculus (either outbred CD-1 or inbred B6) (Fig 3D-F). For example, IRI/contralateral kidney weight ratios at $14 \mathrm{~d}$ were maintained in $A$. cahirinus $(0.92 \pm 0.02)$ compared to almost $40 \%$ loss of renal parenchymal mass to fibrosis in M. musculus (Fig 3D). Likewise, fibrosis severity measured by picrosirius red staining indicated a near total absence of fibrosis in $A$. cahirinus compared to either CD-1 or B6 strains of M. musculus (Fig 3E,F).

To assess the degree of functional restoration in the uni-IRI damaged kidney, we performed uniIRI followed by a contralateral nephrectomy at day 14 and then measured kidney function over the next 2 days. Importantly, we found striking and reproducible differences in 16d BUN levels between A. cahirinus and M. musculus (Fig 3G). Consistent with a near complete absence of tubular damage and interstitial fibrosis at $14 \mathrm{~d}$, we found that BUN levels were nearly normal in A. cahirinus indicative of almost complete restoration of kidney function by $16 \mathrm{~d}$ after IRI (compare Fig 3A to Fig 3G). By contrast, M. musculus BUN levels were substantially increased indicative of progressive renal failure (Fig 3G). Likewise, staining for the tubular epithelial cell 
basement membrane protein laminin showed progressive disruption and thickening by $14 \mathrm{~d}$ in $M$. musculus kidney (B6) consistent with tubular atrophy while basement membrane structures at 14d in A. cahirinus kidney strongly resembled normal uninjured kidney (Ctrl) (Fig $\mathbf{3 H})$ consistent with our Sirius red results (Fig 3E). The removal of necrotic and cellular debris is an important precursor in wound repair and tissue regeneration. ${ }^{2}$ Intraluminal casts and debris were quantitated from kidney sections and showed that both species exhibited abundant casts/debris at equivalent levels by $24 \mathrm{~h}$ after IRI (Fig 4A,B,C, arrows). At later time points, M. musculus retained these intraluminal debris while $A$. cahirinus efficiently cleared them from the tubular network (Fig 4A, D-G). Histologic examination by periodic acid Schiff (PAS) stains demonstrated that there were similar levels of tubular necrosis and tubular casts seen in the corticomedullary junction at $24 \mathrm{~h}$ after severe IRI in both $A$. cahirinus and M. musculus (Supp Fig 3A-C). However, what was strikingly different in $A$. cahirinus was the abundance of polymorphonuclear cells and other nucleated cells within intraluminal tubular casts (Supp Fig 3D,E, arrows) that was seen much less frequently in M. musculus (Supp Fig 3B, arrow). By $72 \mathrm{~h}$ after IRI, tubular casts, dilation, and interstitial inflammation progressed in M. musculus (Supp Fig 3F,G) while in A. cahirinus the intraluminal cellular debris had been removed and replaced by highly nuclear, somewhat disorganized tubular structures (Supp Fig $\mathbf{3 H}$ ) with flattened epithelial cells suggesting progression toward a more dedifferentiated state (Supp Fig $\mathbf{3} \mathbf{3} \mathbf{J}$, arrows). By $7 \mathrm{~d}$, monocytic infiltrates, tubular damage and interstitial fibrosis continue to progress in M. musculus compared to the appearance of defined tubular structures with open lumens in $A$. cahirinus (Supp Fig $3 \mathbf{M}, \mathbf{N}$ ) and PAS-positive brush border structures signifying mature differentiated tubular epithelial cells (Supp Fig 30). These findings confirm that the response to severe acute kidney injury in $A$. cahirinus does not lead to the progressive, degenerative fibrotic response characteristic of $M$. musculus and human kidneys, but instead results in near complete restoration of nephron structure and function. 


\section{Discussion}

Using two different and highly aggressive forms of experimental kidney injury, we show that there was a near complete absence of interstitial renal fibrosis and a remarkable restoration of kidney function in $A$. cahirinus compared to either inbred (B6) or outbred (CD-1) strains of $M$. musculus. These remarkable differences in wound healing responses were not due to the failure of our injury models to produce acute tissue damage in $A$. cahirinus kidneys since histological and functional assays showed equivalent tissue injuries in the first $24-72 \mathrm{~h}$ after UUO or IRI surgeries in both species. Particularly striking was the almost complete restoration of normal kidney function, assessed by blood urea nitrogen levels, by $14 \mathrm{~d}$ after severe ischemiareperfusion injury in $A$. cahirinus (Fig 3G). By contrast, in parallel experiments with $M$. musculus, injured kidneys were severely fibrotic and progressing rapidly towards complete kidney failure (Fig 3G).

Muroid rodents of the genus Acomys (Spiny mice) have evolved the ability to shed their dorsal skin to avoid predation and then to completely regenerate the lost skin tissues without fibrosis or scar formation (4-6). Mechanical and histological assays showed that Acomys skin is specialized to be structurally fragile and prone to tear under low tensile forces. ${ }^{4}$ Therefore it cannot be assumed that the regenerative response to tissue injury in the skin, the first target of predatory attacks in the wild, necessarily extends to internal organs in Acomys species. A similar ear skin regenerative response was previously reported for the MRL/MpJ strain of mice. ${ }^{14}$ However, multiple attempts to determine if regenerative wound healing extended to internal organs, including kidneys, of these mice were generally negative. ${ }^{15-17}$ Thus, our results on the striking absence of fibrotic tissue formation in $A$. cahirinus kidney suggest that the regenerative wound healing response previously described in the $\operatorname{skin}^{4-6}$ may indeed be a systemic property that extends to critical internal organs in this species. 
Acute kidney injury (AKI) initiates a fibrogenic cascade that leaves patients at high risk for developing chronic kidney disease and progressive loss of renal function. ${ }^{18-20}$ Although elegant studies in $M$. musculus have produced substantial insights into the pathogenesis of renal fibrosis, translating these findings into therapeutic solutions has been poor. Wound healing in most adult mammals, including humans, is a process of repair that ultimately replaces functional tissue with a collagen-rich extracellular matrix resulting in a corresponding loss of organ function. By contrast, some fish and amphibian species can fully regenerate tissue damage and restore organ function after amputation or severe tissue injuries. ${ }^{21}$ In the zebrafish kidney, for example, there is evidence of formation of new nephrons after gentamicin nephrotoxicity. ${ }^{22}$ However, in adult mammals there are no reports of nephron formation de novo after kidney injury. We now provide evidence for a potentially transformative new mammalian model for kidney disease that has evolved a distinctly different wound healing response to kidney injury than the currently studied mouse, rat, or human, models. If confirmed for other organs, an indepth analysis of the molecular basis for scar-free wound healing in Acomys species could be a gateway for novel anti-fibrotic therapies.

While our results demonstrating nearly complete restoration of kidney function after severe IRI injury (Fig 3G) are consistent with the likelihood there is nephron regeneration in $A$. cahirinus, they are not currently sufficient to prove this. In models of true vertebrate regeneration, such as axolotl limb, ${ }^{23}$ zebrafish heart, ${ }^{24}$ or Acomys skin, ${ }^{5}$ tissue mass is removed by surgical resection and the lost tissue is fully restored both structurally and functionally. Although tissue damage produced by 40 minutes of renal ischemia/reperfusion is extensive (Fig 3A-C, Fig 4) and restoration of kidney function is remarkably robust (Fig $\mathbf{3 G}$ ), further work is required to definitively establish whether new nephrons are being formed in this model. The most robust phenotypic difference between $A$. cahirinus and $M$. musculus in our study is the near complete absence of fibrotic tissue formed in injured $A$. cahirinus kidneys after either UUO or IRI 
procedures. The extent to which lack of fibrosis is sufficient to explain the differences in wound healing outcomes reported here, or whether other changes in the $A$. cahirinus genome are essential for regenerative wound repair, are important questions for future work.

\section{Figure Legends}

Figure 1. Absence of fibrosis after severe obstructive injury in $A$. cahirinus. $(A, B)(U p p e r)$

The ureter from the left kidney was ligated (arrow) to produce obstructive injury in both $A$. cahirinus and C57BL/6J (B6) mice. (Lower) Upon gross inspection, contralateral kidneys (NK) from the two species are similar in length but $A$. cahirinus UUO kidneys appear less damaged than B6 UUO kidneys after 14d of obstruction; (C) Left panel demonstrates preservation of parenchymal thickness (greater distance between arrows) in A. cahirinus kidney with renal pelvis noted by dotted white line. The graph demonstrates the best fit line of the ratio of UUO:NK kidneys for each time point and the slopes were analyzed by linear regression $(n=6$ 10/time point); (D) Total collagen content was measured by micrograms hydroxyproline per mg wet kidney weight. Graph summarizes total collagen measurement for B6 and A. cahirinus NK and UUO kidneys, (n=6-8/time point for each group); (E) Graph summarizes image analysis of picrosirius red staining for each UUO time point ( $n=6 /$ time point for each group); (F) Masson trichrome sections were blindly scored for IFTA and inflammation (see Methods, $\mathrm{n}=6 /$ time point for each group); Graph summarizes IFTA scores (fibrosis severity score) for each time point; (G) UUO was performed on outbred CD-1 mice (green)(n=3-4/time point) and the development of fibrosis was compared to B6 (blue) and A. cahirinus (red). Graph summarizes image analysis of picrosirius red staining with $\mathbf{( H )}$ representative digital images (400x). Glomeruli are outlined (dotted green). Arrows demonstrate Sirius red staining of interstitial matrix. (I) Dilated tubular area was measured in Masson trichrome sections; Graph summarizes image analysis of tubular dilation area in B6 and $A$. cahirinus after UUO (n=6-7/time point for each group): $B 6$ vs $A$. 
cahirinus: ${ }^{\star} \mathrm{p}<0.05,{ }^{* \star} \mathrm{p}<0.01,{ }^{* \star} \mathrm{p}<0.001,{ }^{* \star *} \mathrm{p}<0.0001 ; C D-1$ vs $A$. cahirinus: $\dagger \mathrm{p}<0.0001 ; B 6$ vs $C D-1: ¥ \mathrm{p}<0.0001)$.

Figure 2. Myofibroblast formation and macrophage infiltration do not generate a fibrotic response in A. cahirinus. (A) Smooth muscle alpha actin (Acta2) expression was investigated by immune-confocal microscopy at days 3, 7, and 14 after UUO. Representative digital images (400x) Acta2 expression (green) for B6 and A. cahirinus at days 7 and 14 after UUO; (B) Graph summarizes image analysis for Acta2 at each time point ( $n=6 /$ time point for each group). (C) F4/80 macrophage infiltration was examined by confocal microscopy and quantified by flow cytometry. Representative digital images (400x) of F4/80 (red) expression at days 7 and 14 after UUO. (D) Flow cytometry was performed on single cell suspensions from whole kidney and analyzed for F4/80 expression. Graph summarizes percent F4/80+ cells at days 3, 7, and 14 after UUO analyzed by FACS ( $n=3-4 /$ time point per group). (E) Tubular integrity was examined by confocal microscopy for Cdh1 (E cadherin). Representative digital images (400x) of Cdh1 (red) expression for B6 and A. cahirinus. (F) Graph summarizes image analysis results for Cdh1 levels at each time point ( $n=6 /$ time point for each group). (B6 (blue) vs. A. cahirinus (red): ${ }^{*} p<0.05,{ }^{* *} p<0.01,{ }^{* \star *} p<0.001,{ }^{* \star *} p<0.0001$; between time points $\left.\# p<0.05, \# \# p<0.01\right)$

Figure 3. Near complete recovery of nephron function after severe ischemic injury in $A$.

cahirinus. (A) B6 and A. cahirinus underwent unilateral IRI (uni-IRI) with contralateral nephrectomy and sacrificed at $24 \mathrm{~h}$. BUN levels were assessed in uninjured animals (Ctrl) and those sacrificed at $24 \mathrm{~h}$ ( $n=4-5 /$ time point per group): ): NS-not significant, * $p<0.05$, ${ }^{* \star} p<0.01$. Note that both species exhibited equivalent severe acute kidney damage in response to ischemic injury. (B) H\&E stained sections were blindly scored for tubular injury (see Methods, n=4-5/group); Representative H\&E digital images (400x) at 24h from IRI kidneys. 
Arrows indicate tubular casts and necrotic cell debris. (C) Graph summarizes tubular injury scores at $24 \mathrm{~h}$ after IRI. (D) In order to determine effect of acute injury on repair, uni-IRI was performed without nephrectomy on B6, CD-1, and A.cahirinus. Graph summarizes data on IRI/contralateral (NK) kidney weight ratio at time of sacrifice at $14 d(n=4-6 / g r o u p): ~ * ~ p<0.05$, ${ }^{\star *} \mathrm{p}<0.01$. (E) Graph summarizes image analysis of picrosirius red staining for each UUO time point ( $n=5-6 /$ time point for each group); (B6 (blue), CD-1 (green), A.cahirinus (red): * $p<0.05$, ${ }^{\star \star \star *} p<0.0001$. (F) Representative picrosirius red digital images (400x). Glomeruli are outlined (dotted green). Arrows demonstrate Sirius red staining of interstitial matrix. (G) In order to determine functional recovery, uni-IRI was performed, the contralateral kidney was removed at day 14 and kidney function was monitored until sacrifice at day 16 . BUN levels were determined in uninjured animals (Ctrl) and those sacrificed at day 16, 2 days after contralateral nephrectomy (IRI). Note the nearly complete recovery of nephron function in the injured $A$. cahirinus kidneys at $16 \mathrm{~d}$ compared to the high BUN levels indicative of kidney failure in B6 injured kidneys: NS-not significant, ${ }^{* * *} \mathrm{p}<0.0001$. (H) Laminin (red) immunostaining of tubular epithelial basement membrane architecture at days 0, 1, and 14 after IRI injury (400x; scale bars 100um). Note $A$. cahirinus kidney at day 14 (D14 IRI) strongly resembles uninjured kidney (Ctrl) in tubular basement membrane architecture, while B6 basement membranes demonstrate collapse and thickening with advancing fibrosis. Scale bars; $100 \mu \mathrm{m}$.

\section{Figure 4. Rapid clearance of tubular debris after severe ischemic injury in $A$.}

cahirinus. Tubular casts and debris were identified on Periodic acid Schiff (PAS) stain in unilateral IRI kidneys at 24h, 72h, and 7 days in B6 and A. cahirinus.. (A) Graph summarizes image analysis results for tubular casts and intraluminal debris after IRI. (B6-blue, A. cahirinusred; $B 6$ vs $A$. cahirinus: $\left.{ }^{\star \star} \mathrm{p}<0.01,{ }^{\star \star \star} \mathrm{p}<0.001\right)$. Representative fields from $200 x$ images demonstrate representative tubular casts and intraluminal debris (arrow) in IRI kidneys at 24h 
$(B, C), 72 h(D, E)$, and 7 days $(F, G)$ in $B 6$ and $A$. cahirinus kidneys. Arrows demonstrate areas of intraluminal debris/casts.

\section{Supplemental Figure Legends}

Supplemental Figure 1. Picrosirius red staining in B6 and A. cahirinus. Representative picrosirius red digital images (400x) in B6 and A. cahirinus after UUO. Glomeruli are outlined (dotted green). Arrows demonstrate Sirius red staining of interstitial matrix.

Supplemental Figure 2. Fibrosis severity score in B6 and A. cahirinus. Masson trichrome sections from were blindly scored for IFTA and inflammation (see Methods, $n=6 /$ time point for each group). Representative Masson trichrome digital images (400x) after UUO.

Supplemental Figure 3. Restoration of nephron structure after severe ischemic injury in A. cahirinus. Periodic acid Schiff (PAS) stain was performed on unilateral IRI kidneys at (A-E) 24h, (F-J) 72h, and (K-O) 7 days in B6 and A. cahirinus. Low power images (100x) in B6 demonstrate progression of $(\mathbf{A})$ necrotic kidney injury to $(\mathbf{F}, \mathbf{K})$ tubular casts and loss of tubular structure with interstitial inflammation. In comparison, low power images in $A$. cahirinus show similar necrotic injury $(\mathbf{C})$, with accelerated repair and restoration of nephron structure $(\mathbf{H}, \mathbf{M})$. At 24h, polymorphonuclear cells can be seen in tubular lumens and casts in B6 (arrow B), and to an even greater extent in $A$. cahirinus (arrows E). At $72 \mathrm{~h}$, tubular casts are nearly resolved (arrow, I), and flattened, dedifferentiated tubular epithelial cells (I) and tubular lumens in repair (arrow $\mathbf{J}$ ) can be seen in $A$. cahirinus. At 7 days, tubular casts and interstitial inflammation remain in B6 (L), compared to PAS positive brush border cells in A. cahirinus (N,O). 


\section{Concise Methods (to be in online supplement)}

\section{Experimental Design}

We utilized two models of kidney injury to investigate the differences in wound healing and fibrosis: Unilateral ureteral obstruction (UUO) and ischemia reperfusion injury (IRI). C57BL/6J (B6) and CD-1 mice were used as inbred and outbred strains of $M$. musculus, respectively. Surgery was performed on male animals between 3 and 6 months of age. UUO surgeries were performed as previously described ${ }^{25,26}$ ( $n=6$-8/group), and animals were sacrificed at $3,7,14$, and 21 days after surgery. Mice received isoflurane anesthesia (5\% induction; 1-3\% maintenance) in oxygen through a precision vaporizer (Portable Anesthesia Machine, PAM; Molecular Imaging Products, Bend, OR, USA).

Unilateral IRI (uni-IRI) surgeries were performed as previously described ${ }^{25,26}$ except that the vascular pedicle was clamped for 40 minutes ( $n=5-6 / g r o u p)$, and animals were sacrificed at $24 \mathrm{~h}$, 72h, 7 days, and 14 days after surgery. In order to assess initial injury, the contralateral kidney was removed at the time of uni-IRI surgery and sacrificed at $24 \mathrm{~h}$ after surgery. In order to quantify kidney function in the uni-IRI kidney after initial injury, a contralateral nephrectomy was performed 14 days post-surgery as previously described ${ }^{25}$ Blood was drawn daily until sacrifice at 2 days post-nephrectomy. All procedures were performed in accordance with the guidelines established by National Research Council Guide for the Care and Use of Laboratory Animals and approval of our Institute Animal Care and Use Committee (IACUC). Contralateral, UUO, and IRI kidneys were harvested and processed for RNA and protein extraction and histological studies as previously described. ${ }^{25-27}$ Frozen tissue samples were stored at- $80^{\circ} \mathrm{C}$.

\section{Collagen content}


Hydroxyproline content of kidney tissue ( $\mu \mathrm{g}$ of hydroxyproline per mg of wet weight kidney section) was measured by acid hydrolysis of the tissue section using procedures established in our laboratory. ${ }^{25-27}$

\section{Histological examination}

Immunohistochemical staining was performed on sections of paraffin-embedded tissue or cryosections of snap-frozen tissue using procedures established in our laboratory with VECTASTAIN Elite ABC Kits (Vector Laboratories, Inc.) and AEC Substrate Chromogen K3464 (Dako Corp.). Sections were blocked with Avidin/Biotin blocking kit (Vector Laboratories, Inc.). Computer-assisted image analysis was performed on 6 randomly selected 400x magnified images of slides from individual animals with Image-Pro Plus software (Mediatech). The investigator was blinded to the experimental groups at the time of analysis. Picrosirius red staining was performed as previously described. ${ }^{25,27}$ In brief, quantification of interstitial staining of picrosirius red (SR) staining was performed in a blinded manner using Image-Pro software with randomly selected cortical fields. SR glomerular staining was subtracted and net SR area was normalized to net tubulointerstitial area of 400x field (Net area $=$ Total - glomerular area empty space). Masson Trichrome and hematoxylin eosin stains were performed on paraffin sections by standard protocols. Interstitial fibrosis and tubular atrophy (IFTA) scores was analyzed on 6 randomly selected 400x Masson Trichrome stained images. The following IFTA scores were assigned, in a blinded manner, based on the estimated percent area affected with tubular atrophy, loss of tubular structure, interstitial inflammation, and interstitial fibrosis in the field: 1 (normal); 2 (<10\%), $3(10-25 \%) ; 4(26-50 \%) ;$ or $5(>50 \%)$. Dilated tubular area was measured using Image-Pro software on 400x Masson trichrome stained images. Tubular casts and intraluminal debris area was measured using Image-Pro software on 200x PAS-stained images and normalized to net tubulointerstitial area for 6 randomly selected cortical fields. 
Secondary antibodies were shown to be non-reactive with tissue sections stained without the primary antibody.

\section{F4/80 macrophage quantification}

Mice were perfused with cold normal saline and contralateral and UUO kidneys were placed on ice, digested with Liberase TL (Roche) with 1\% DNase (Sigma-Aldrich), then placed at $37^{\circ} \mathrm{C}$ for 10 minutes, as previously described. Glomeruli were removed by passing cell suspension through a $40 \mu \mathrm{m}$ Nylon filter. Cells were stained per protocol with DAPI, PE-Cy7-anti-CD45, PEanti-CD11b, APC-eFluor780-F4/80 from BD Sciences. Cells were blocked with mouse Fc Block (BD Biosciences). Leukocytes were identified and gated based on their positive F4/80 expression. Data was acquired on the LSR II flow cytometer (BD Biosciences) and analyzed using FlowJo software (Tree Star, Inc).

\section{Kidney function}

Serum was analyzed for blood urea nitrogen (BUN) using the Urea Nitrogen (BUN) Reagent Set kit (Teco Diagnostics). Samples were processed according to manufacturer's protocol. All samples were performed in triplicate.

\section{Immunofluoresence}

For cryosectioning, excised tissue was embedded and flash-frozen in O.C.T medium (Tissue Tech) using a dry-ice slurry/2-methylbutanol mixture and cryosectioned between 8-10um. Tissue cryosections were washied with PBS and fixed with 4\% PFA for 10 min. Post fixation, slides were washed three times for 5 min each with PBS followed by permeabilization using 0.2\% Triton-X100 in PBS (PBT) for 10 min. Slides were then blocked (5\% BSA, 2\% normal goat serum in PBT) at room temperature for $1 \mathrm{hr}$. Post block, tissue sections were then incubated in primary antibody overnight at $4 \mathrm{C}$ in blocking solution (3\% BSA, $0.2 \%$ Triton-X100 
in PBS). Primary antibodies used include pan-Laminin (Abcam, \#ab11575), Acta2 (Sigma, \#A2547), F4/80 (Invitrogen, \#MF48020), and Cdh1 (BD Bioscience, \#610181). After overnight incubation, slides were washed with PBS, and then incubated with goat ALEXA-Fluor 594- or ALEXA-Fluor 488- conjugated antibodies (Thermo Fisher Scientific) for $2 \mathrm{~h}$ at room temperature in blocking solution. Cell nuclei were counterstained with DAPI (Molecular Probes) and mounted in 4\% (w/v) propyl gallate anti-fade solution. Immunofluorescent images were obtained using an SP5 confocal microscope (Leica). Acta2 and Cdh1 confocal image analysis was performed as previously described..$^{25,27}$

\section{Statistical analysis}

All data are presented as the mean and standard error. All statistical analyses were performed using GraphPad PRISM 7.0 (GraphPad Software) and STATA 14 (StataCorp LP) software. Two-way analysis of variance (ANOVA) was performed for all parametric data including computer-assisted image analysis data for time and species. For image analysis data, the arithmetic mean of six randomly selected images of slides for each animal was used for the twoway ANOVA. Sidak's and Tukey's multiple comparison post-tests were utilized for time and species, respectively. Nonparametric data (IFTA and tubular injury scores) was analyzed using the Mann-Whitney $U$ test. A $P$ value $<0.05$ was considered statistically significant. UUO kidney weights were analyzed by linear regression. 


\section{References}

1. Duffield, J.S. et al. Host responses in tissue repair and fibrosis. Annu. Rev. Pathol. 8, 241-276 (2013).

2. Gurtner, G.C. et al. Wound repair and regeneration. Nature 453, 314-321 (2008).

3. Humphreys, B.D. Mechanisms of renal fibrosis. Annu. Rev. Physiol. 80, (2018).

4. Seifert, A.W. et al. Skin shedding and tissue regeneration in African spiny mice (Acomys). Nature 489, 561-565 (2012).

5. Gawriluk, T.R. et al. Comparative analysis of ear-hole closure identifies epimorphic regeneration as a discrete trait in mammals. Nat. Commun. 7, 11164 (2016).

6. Matias Santos, D. et al. Ear wound regeneration in the African spiny mouse Acomys cahirinus. Regeneration (Oxf) 3, 52-61 (2016).

7. Duffield, J.S. Cellular and molecular mechanisms in kidney fibrosis. J. Clin. Invest. 124, 2299-2306 (2014).

8. Tapmeier, T.T. et al. Reimplantation of the ureter after unilateral ureteral obstruction provides a model that allows functional evaluation. Kidney Int. 73, 885-889 (2008).

9. Liu, J. et al. Cell-specific translational profiling in acute kidney injury. J. Clin. Invest. 124, 1242-1254 (2014).

10. Lin, S.L. et al. Bone marrow Ly6Chigh monocytes are selectively recruited to injured kidney and differentiate into functionally distinct populations. J. Immunol. 183, 67336743 (2009).

11. Liu, W. et al. Dragon (repulsive guidance moledule GGMb) inhibits E-cadherin expression and induces apoptosis in renal tubular epithelial cells. J. Biol. Chem. 288, 31528-31539 (2013).

12. Chaabane, W. et al. Renal functional decline and glomerulotubular injury are arrested but not restored by release of unilateral ureteral obstruction (UUO). Am. J. Physiol. Renal Physiol. 304, F432-F439 (2013). 
13. Zheng, G. et al. Alpha3 integrin of cell-cell contact mediates kidney fibrosis by integrinlinked kinase in proximal tubular E-cadherin-deficient mice. Am. J. Pathol. 186,18471860 (2016).

14. Leferovich, J.M. et al. Heart regeneration in adult MRL mice. Proc. Natl. Acad. Sci. USA 98, 9830-9835 (2001).

15. Robey, T.E., Murry, C.E. Absence of regeneration in the MRL/MpJ mouse heart following infarction or cryoinjury. Cardiovasc. Pathol. 17, 6-13 (2008).

16. Oh, Y.S. et al. Scar formation after ischemic myocardial injury in MRL mice. Cardiovasc. Pathol. 13, 203-206 (2004).

17. Iwata, T. et al. Aberrant macrophages mediate defective kidney repair that triggers nephritis in lupus-susceptible mice. J. Immunol. 188, 4568-4580 (2012).

18. Chawla, L.S., et al. Acute kidney injury and chronic kidney disease as interconnected syndromes. New Engl. J. Med. 371, 58-66 (2014).

19. Ferenbach DA, Bonventre JV. Mechanisms of maladaptive repair after AKI leading to accelerated kidney ageing and CKD. Nat. Rev. Nephrol. 11, 264-276 (2015).

20. Liu, J. et al. Molecular characterization of the transition from acute to chronic kidney injury following ischemia/reperfusion. JCI Insight 2, e94716 (2017).

21. Poss, K.D. Advances in understanding tissue regenerative capacity and mechanisms in animals. Nat. Rev. Genet. 11, 710-722 (2010).

22. McCampbell, K.K., Wingert, R.A. New tides: using zebrafish to study renal regeneration. Transl. Res. 163, 109-122 (2014).

23. Kragl, M. et al. Cells keep a memory of their tissue origin during axolotl limb regeneration. Nature 460, 60-65 (2009).

24. Kikuchi, K. et al. Primary contribution to zebrafish heart regeneration by gata4(+) cardiomyocytes. Nature 464, 601-605 (2010). 
25. Pennathur, S. et al. The macrophage phagocytic receptor CD36 fibrogenic pathways on removal of apoptotic cells during chronic kidney injury. Am J Pathol 185, 2232-2245 (2015).

26. Okamura, DM. et al. Cysteamine modulates oxidative stress and blocks myofibroblast activity in CKD. J Am Soc Nephrol 25, 43-54 (2014).

27. Okamura, DM. et al. CD36 regulates oxidative stress and inflammation in hypercholesterolemic CKD. J Am Soc Nephrol 20, 495-505 (2009). 


\section{Acknowledgments}

This work was supported by a grant from the W.M. Keck Foundation, the US National Institutes of Health grant 1R21OD-023838, the Loie Power Robinson Stem Cell \& Regenerative Medicine Fund, and the Seattle Children's Research Institute.

\section{Author Contributions}

DMO, CMB, PW, NB, KB, AT, XS, JO, AMP, BRN performed the experiments. DMO, DRB, MWM designed the experiments. DMO, CMB, MWM wrote the manuscript. DMO, KJM, MWM obtained funding for the project. All authors made critical input into editing the manuscript.

\section{Author Information}

Correspondence and requests for materials should be addressed to one of the following: DO (daryl.okamura@seattlechildrens.org); KJM (kathleen.millen@seattlechildrens.org); or MWM (mwm84@uw.edu). 


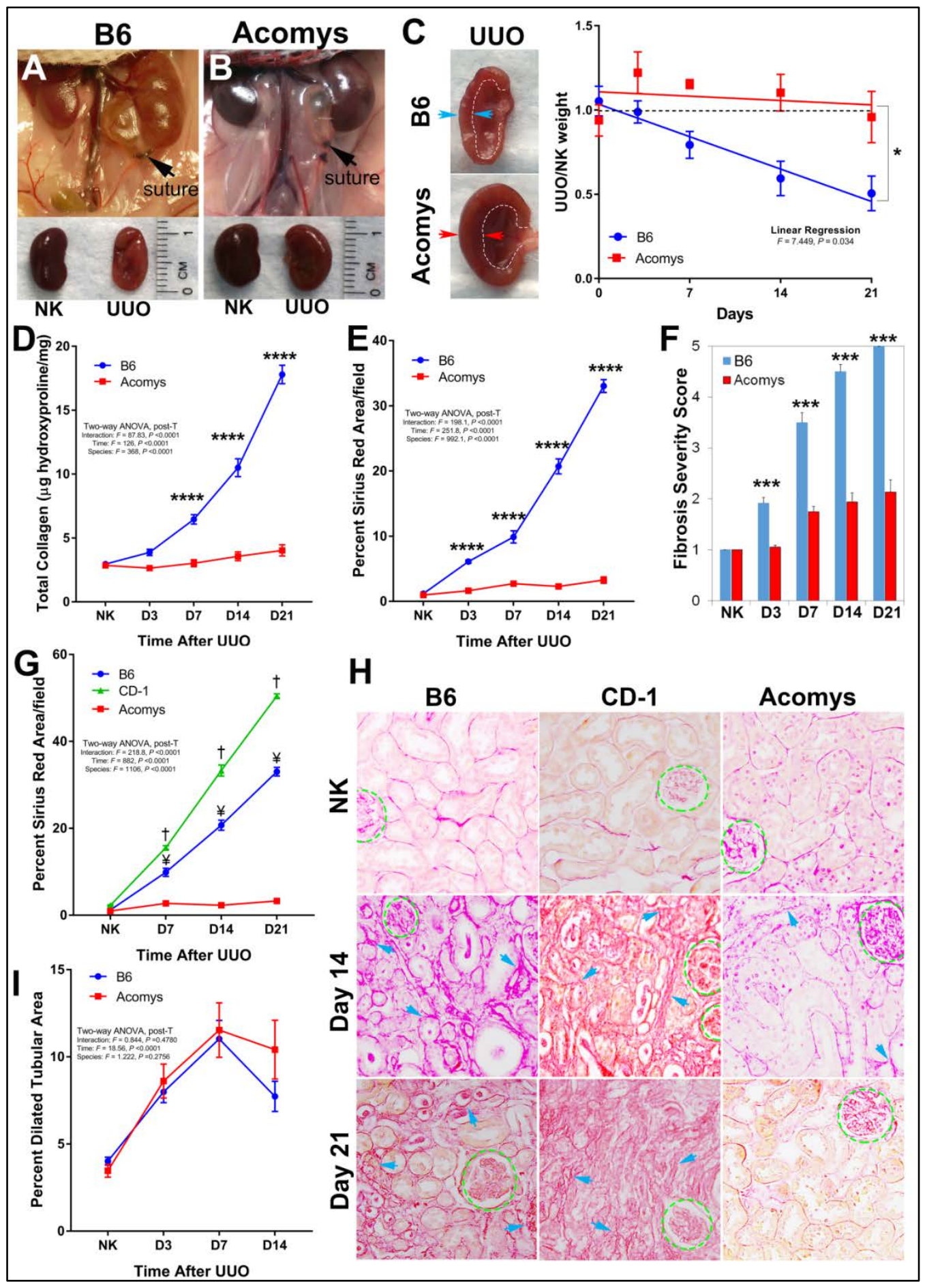

Figure 1. Absence of fibrosis after severe obstructive injury in $\mathbf{A}$. cahirinus. $(\mathbf{A}, \mathbf{B})($ Upper) The ureter from the left kidney was ligated (arrow) to produce obstructive injury in both $A$. cahirinus and C57BL/6J (B6) mice. (Lower) Upon gross inspection, contralateral kidneys (NK) from the two species are similar in length but $A$. cahirinus UUO kidneys appear less damaged than B6 UUO kidneys after $14 \mathrm{~d}$ of obstruction; (C) Left panel demonstrates preservation of parenchymal thickness (greater distance between arrows) in $A$.

cahirinus kidney with renal pelvis noted by dotted white line. The graph demonstrates the best fit line of the ratio of UUO:NK kidneys for each time point and the slopes were analyzed by linear regression ( $n=6-10 /$ time point); (D) Total collagen content was measured by micrograms hydroxyproline per mg wet kidney weight. Graph summarizes total collagen measurement for B6 and $A$. cahirinus NK and UUO kidneys, ( $n=6-8 /$ time point for each group); (E) Graph summarizes image analysis of picrosirius red staining for each UUO time point ( $\mathrm{n}=6 /$ time point for each group); (F) Masson trichrome sections were blindly scored for IFTA and inflammation (see Methods, n=6/time point for each group); Graph summarizes IFTA scores (fibrosis severity score) for each time point; (G) UUO was performed on outbred CD-1 mice (green)(n=3-4/time point) and the development of fibrosis was compared to B6 (blue) and A. cahirinus (red). Graph summarizes image analysis of picrosirius red staining with $\mathbf{H}$ ) representative digital images (400x). Glomeruli are outlined (dotted green). Arrows demonstrate Sirius red staining of interstitial matrix. (I) Dilated tubular area was measured in Masson trichrome sections; Graph summarizes image analysis of tubular dilation area in B6 and $A$. cahirinus after UUO ( $\mathrm{n}=6-7 /$ time point for each group): $B 6$ vs $A$. cahirinus: * $p<0.05,{ }^{\star \star} p<0.01$, ${ }^{\star \star \star} p<0.001$, ${ }^{\star \star \star \star} p<0.0001 ; C D-1$ vs $A$. cahirinus: $\dagger p<0.0001 ; B 6$ vs $C D-1: ¥ p<0.0001$ ). 


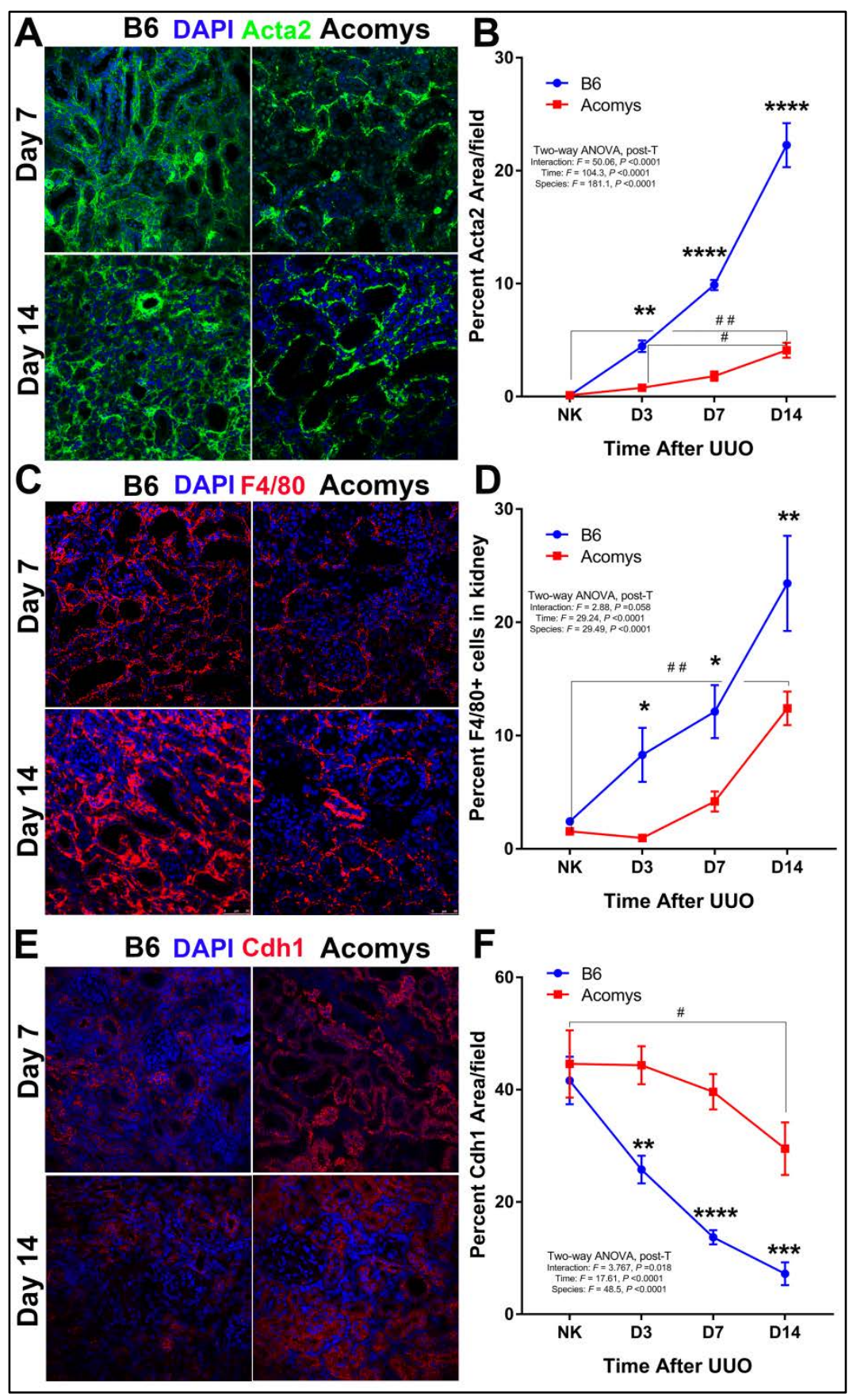

Figure 2. Myofibroblast formation and macrophage infiltration do not generate a fibrotic response in $A$. cahirinus. (A) Smooth muscle alpha actin (Acta2) expression was investigated by immune-confocal microscopy at days 3, 7, and 14 after UUO.

Representative digital images (400x) Acta2 expression (green) for B6 and A. cahirinus at days 7 and 14 after UUO; (B) Graph summarizes image analysis for Acta2 at each time point ( $n=6 /$ time point for each group). (C) F4/80 macrophage infiltration was examined by confocal microscopy and quantified by flow cytometry. Representative digital images (400x) of F4/80 (red) expression at days 7 and 14 after UUO. (D) Flow cytometry was performed on single cell suspensions from whole kidney and analyzed for F4/80

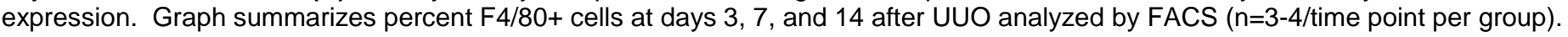
(E) Tubular integrity was examined by confocal microscopy for Cdh1 (E cadherin). Representative digital images (400x) of Cdh1 (red) expression for B6 and A. cahirinus. (F) Graph summarizes image analysis results for Cdh1 levels at each time point ( $\mathrm{n}=6 /$ time point for

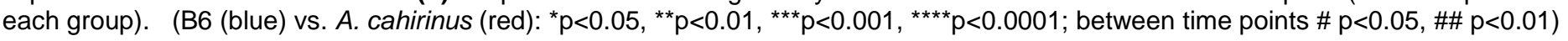




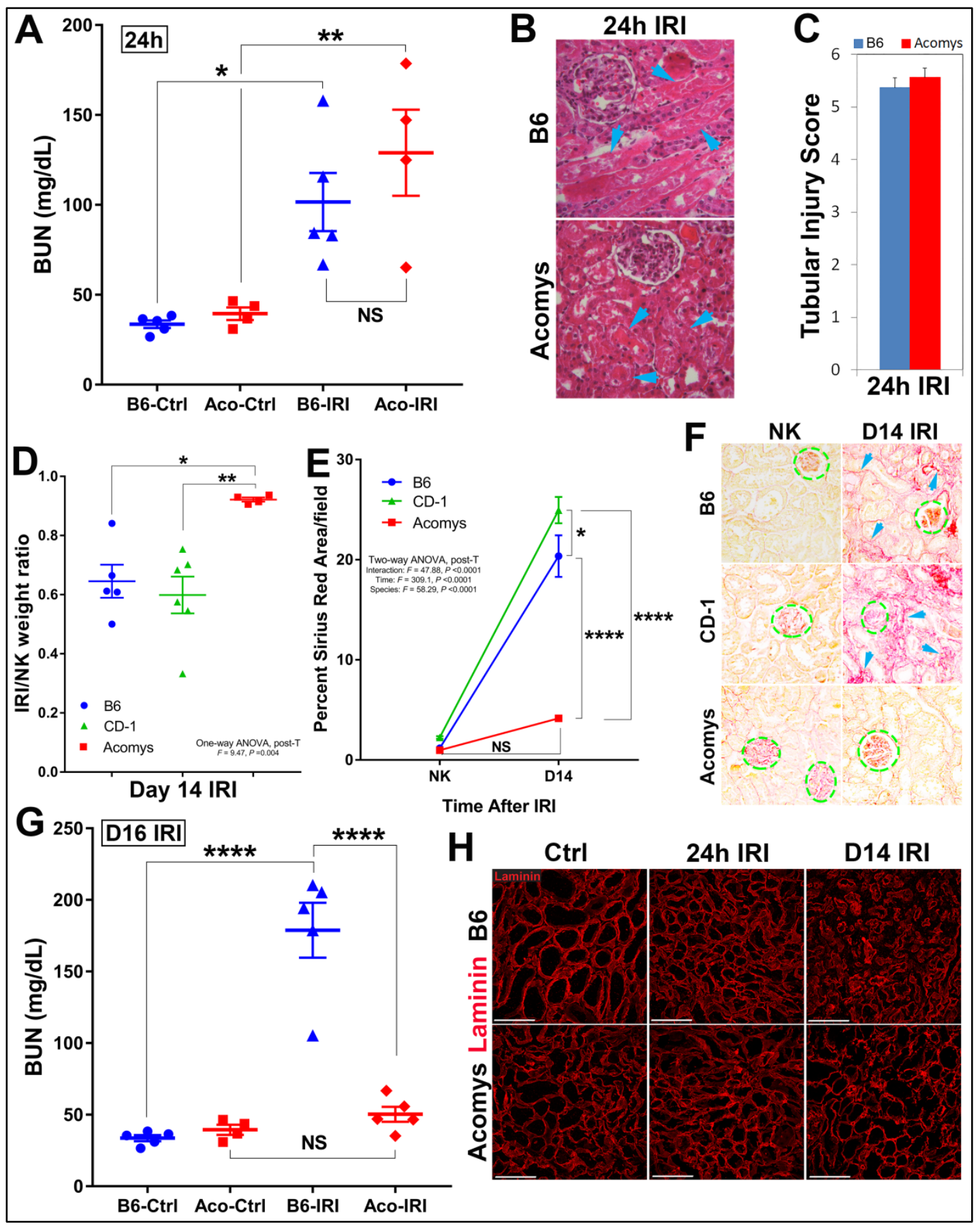

Figure 3. Near complete recovery of nephron function after severe ischemic injury in A. cahirinus. (A) B6 and $A$. cahirinus underwent unilateral IRI (uni-IRI) with contralateral nephrectomy and sacrificed at $24 \mathrm{~h}$. BUN levels were assessed in uninjured animals (Ctrl) and those sacrificed at $24 \mathrm{~h}$ ( $\mathrm{n}=4-5 /$ time point per group): ): NS-not significant, * $\mathrm{p}<0.05,{ }^{*} \mathrm{p}<0.01$. Note that both species exhibited equivalent severe acute kidney damage in response to ischemic injury. (B) H\&E stained sections were blindly scored for tubular injury (see Methods, n=4-5/group); Representative H\&E digital images (400x) at 24h from IRI kidneys. Arrows indicate tubular casts and necrotic cell debris. (C) Graph summarizes tubular injury scores at $24 \mathrm{~h}$ after IRI. (D) In order to determine effect of acute injury on repair, uni-IRI was performed without nephrectomy on B6, CD-1, and A.cahirinus. Graph summarizes data on IRI/contralateral

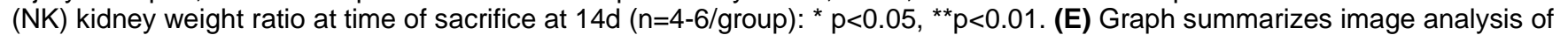
picrosirius red staining for each UUO time point ( $n=5-6 /$ time point for each group); (B6 (blue), CD-1 (green), A.cahirinus (red): * $p<0.05$, ${ }_{\star \star \star \star \star} p<0.0001$. (F) Representative picrosirius red digital images (400x). Glomeruli are outlined (dotted green). Arrows demonstrate Sirius red staining of interstitial matrix. $(\mathbf{G})$ In order to determine functional recovery, uni-IRI was performed, the contralateral kidney was removed at day 14 and kidney function was monitored until sacrifice at day 16. BUN levels were determined in uninjured animals (Ctrl) and those sacrificed at day 16, 2 days after contralateral nephrectomy (IRI). Note the nearly complete recovery of nephron function in the injured $A$. cahirinus kidneys at 16d compared to the high BUN levels indicative of kidney failure in B6 injured kidneys: NS-not significant, ${ }^{\star \star \star *} p<0.0001$. (H) Laminin (red) immunostaining of tubular epithelial basement membrane architecture at days 0,1 , and 14 after IRI injury (400x; scale bars 100um). Note $A$. cahirinus kidney at day 14 (D14 IRI) strongly resembles uninjured kidney (Ctrl) in tubular basement membrane architecture, while B6 basement membranes demonstrate collapse and thickening with advancing fibrosis. Scale bars; $100 \mu \mathrm{m}$. 


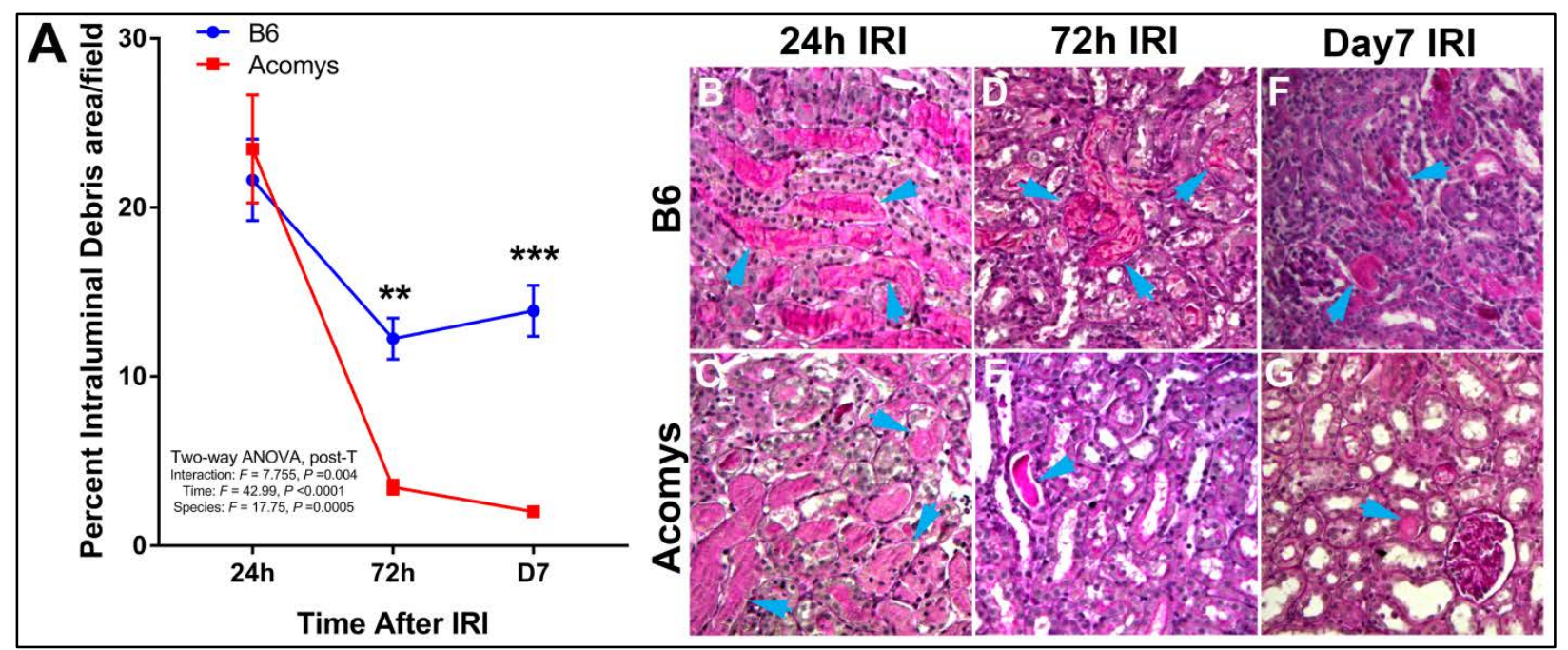

Figure 4. Rapid clearance of tubular debris after severe ischemic injury in $\boldsymbol{A}$. cahirinus. Tubular casts and debris were identified on Periodic acid Schiff (PAS) stain in unilateral IRI kidneys at 24h, $72 \mathrm{~h}$, and 7 days in B6 and A. cahirinus.. (A) Graph summarizes image analysis results for tubular casts and intraluminal debris after IRI. (B6-blue, A. cahirinus-red; B6 vs A. cahirinus: ${ }^{\star *} \mathrm{p}<0.01$, $\star \star \star x<0.001$ ). Representative fields from 200x images demonstrate representative tubular casts and intraluminal debris (arrow) in IRI kidneys at $24 \mathrm{~h}(\mathbf{B}, \mathbf{C}), 72 \mathrm{~h}(\mathbf{D}, \mathrm{E})$, and 7 days $(\mathbf{F}, \mathbf{G})$ in $\mathrm{B} 6$ and $A$. cahirinus kidneys. Arrows demonstrate areas of intraluminal debris/casts. 
bioRxiv preprint doi: https://doi.org/10.1101/315069; this version posted May 4, 2018. The copyright holder for this preprint (which was not certified by peer review) is the author/funder. All rights reserved. No reuse allowed without permission.

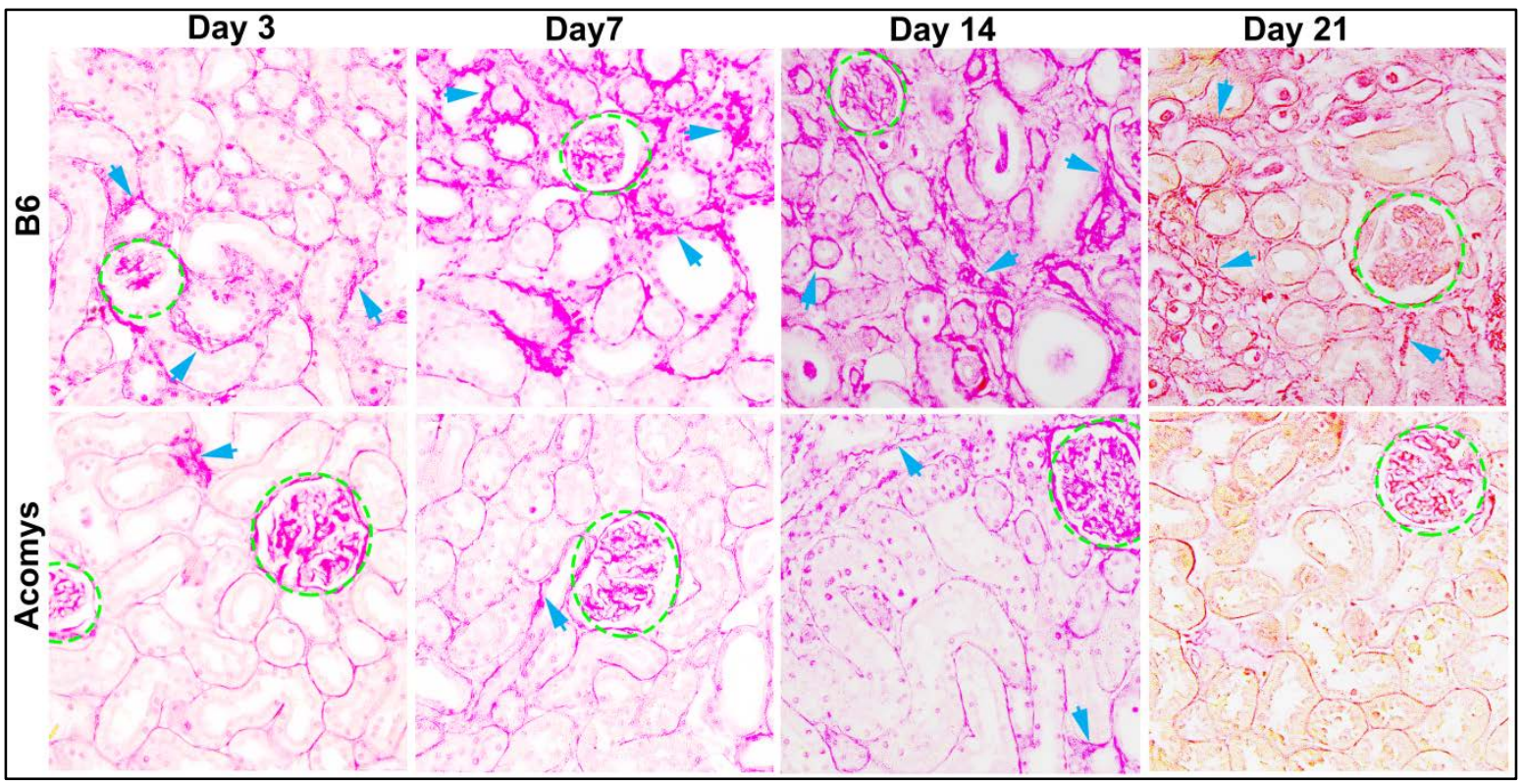

Supplemental Figure 1. Picrosirius red staining in $\mathbf{B 6}$ and $\boldsymbol{A}$. cahirinus. Representative picrosirius red digital images (400x) in B6 and $A$. cahirinus after UUO. Glomeruli are outlined (dotted green). Arrows demonstrate Sirius red staining of interstitial matrix.

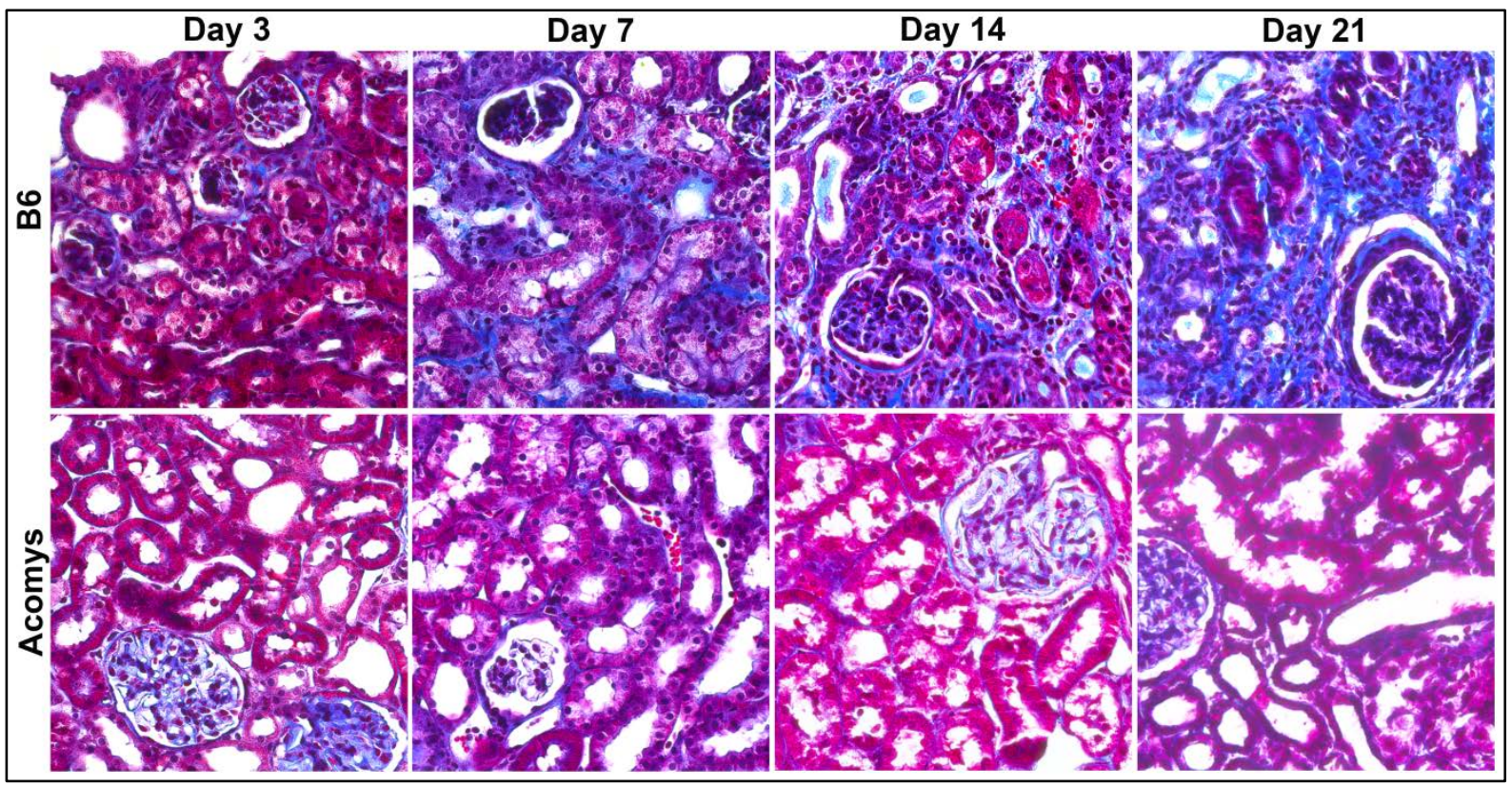

Supplemental Figure 2. Fibrosis severity score in B6 and A. cahirinus. Masson trichrome sections from were blindly scored for IFTA and inflammation (see Methods, $n=6 /$ time point for each group). Representative Masson trichrome digital images (400x) after UUO. 
bioRxiv preprint doi: https://doi.org/10.1101/315069; this version posted May 4, 2018. The copyright holder for this preprint (which was not certified by peer review) is the author/funder. All rights reserved. No reuse allowed without permission.

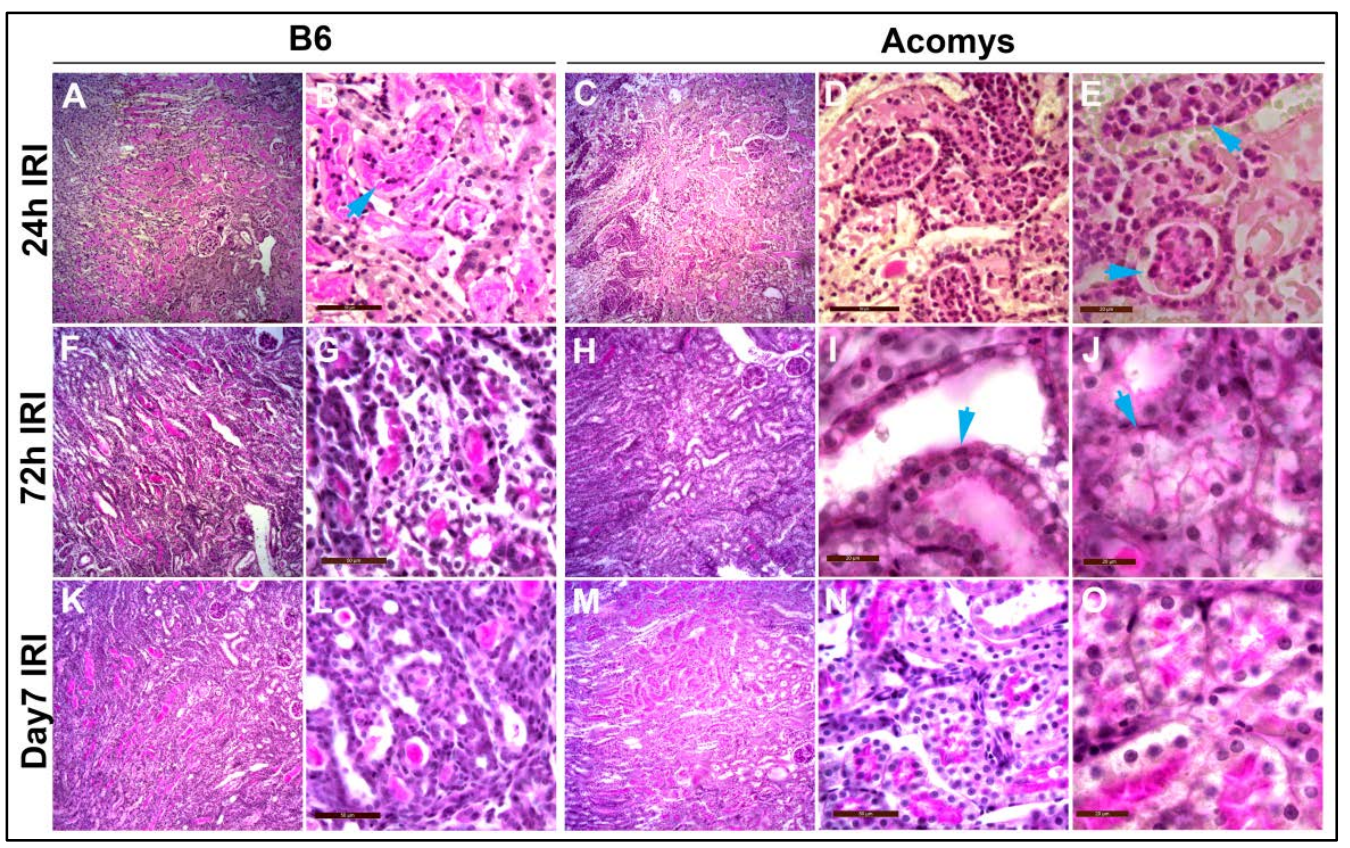

Supplemental Figure 3. Restoration of nephron structure after severe ischemic injury in $A$. cahirinus. Periodic acid Schiff (PAS) stain was performed on unilateral IRI kidneys at (A-E) $24 \mathrm{~h}$, (F-J) $72 \mathrm{~h}$, and (K-O) 7 days in B6 and $A$. cahirinus. Low power images (100x) in B6 demonstrate progression of $(\mathbf{A})$ necrotic kidney injury to $(\mathbf{F}, \mathbf{K})$ tubular casts and loss of tubular structure with interstitial inflammation. In comparison, low power images in Acomys show similar necrotic injury (C), with accelerated repair and restoration of nephron structure $(\mathbf{H}, \mathbf{M})$. At $24 \mathrm{~h}$, polymorphonuclear cells can be seen in tubular lumens and casts in B6 (arrow B), and to an even greater extent in A. cahirinus (arrows E). At 72h, tubular casts are nearly resolved (arrow, I), and flattened, dedifferentiated tubular epithelial cells (I) and tubular lumens in repair (arrow $\mathbf{J}$ ) can be seen in $A$. cahirinus. At 7 days, tubular casts and interstitial inflammation remain in B6 (L), compared to PAS positive brush border cells in A. cahirinus $(\mathbf{N}, \mathbf{O})$. 